\title{
A novel machine-learning-derived genetic score correlates with measurable residual disease and is highly predictive of outcome in acute myeloid leukemia with mutated NPM1
}

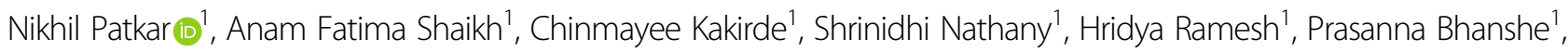 \\ Swapnali Joshi', Shruti Chaudhary', Sadhana Kannan², Syed Hasan Khizer', Gaurav Chatterjee ${ }^{1}$, Prashant Tembhare ${ }^{1}$, \\ Dhanalaxmi Shetty ${ }^{4}$, Anant Gokarn ${ }^{3}$, Sachin Punatkar ${ }^{3}$, Avinash Bonda ${ }^{3}$, Lingaraj Nayak ${ }^{3}$, Hasmukh Jain ${ }^{3}$, Navin Khattry ${ }^{3}$, \\ Bhausaheb Bagal ${ }^{3}$, Manju Sengar ${ }^{3}$, Sumeet Gujral ${ }^{1}$ and Papagudi Subramanian ${ }^{1}$
}

Dear Editor,

Acute myeloid leukemia with mutated NPM1 $\left(N P M 1^{\text {mut }}\right.$ AML), one of the commonest subtypes of AML, is characterized by a favorable outcome in the absence of accompanying FLT3-internal tandem duplications $(\mathrm{ITD})^{1}$. NPM1 ${ }^{\mathrm{mut}}$ AML has a high degree of mutational heterogeneity and harbors an average of 3-4 mutations per case (most commonly involving genes implicated in DNA methylation, cell signaling, cohesin complex, and RNA splicing) ${ }^{2}$. Due to advances in sequencing technologies, we now recognize that agerelated clonal hematopoiesis (ARCH) is a possible precursor to myeloid malignancies, such as myelodysplastic syndromes and AML. Mutations in genes such as DNMT3A, TET2, and ASXL1 account for $>90 \%$ of ARCH mutations in $\mathrm{AML}^{3}$. Interestingly, mutations in these ARCH-defining genes are also harbored by $N P M 1^{\text {mut }}$ AML indicating a putative synergistic mechanism in contributing to leukemogenesis ${ }^{2}$. In that context, variant allele fractions (VAF) generated through next-generation sequencing (NGS) data sets are informative in recreating clonal hierarchy of a tumor sample. By using this information, we can distinguish founder mutations (which would have a higher VAF) from sub-clonal mutations that arise subsequently ${ }^{4}$.

\footnotetext{
Correspondence: Nikhil Patkar (nvpatkar@gmail.com)

${ }^{1}$ Haematopathology Laboratory, ACTREC, Tata Memorial Centre, Navi Mumbai, Maharashtra, India

${ }^{2}$ Biostatistics, ACTREC, Tata Memorial Centre, Navi Mumbai, Maharashtra, India Full list of author information is available at the end of the article.
}

Although NGS technologies have produced a deluge of cancer genomics data, it is challenging to accurately predict disease outcome from these data sets. Machine learning (ML), a branch of artificial intelligence, has shown tremendous potential toward interpretation of complex genomic data sets ${ }^{5}$. By using ML, researchers are now able to discover novel patterns between data and use this information for predicting cancer susceptibility, recurrence, prognostication, and therapy ${ }^{6}$. In addition, ML has also been used to predict transplant-related mortality with considerable success ${ }^{7}$. In a proof of concept, we used a supervised ML approach to identify clinically important genomic aberrations in $N P M 1^{\text {mut }}$ AML. Based on these data, we developed a scoring model that provides a mechanism to risk stratify $N P M 1^{\text {mut }} \mathrm{AML}$, a seemingly homogeneous disease entity.

A total of 110 patients (Supplementary Table 1) of adult ( $\geq 18$ years) $N P M 1^{\text {mut }}$ AML were accrued over a 6-year period from March 2012 to December 2018. The median follow-up for our cohort was 26.8 months. The mean OS was 46.7 months (median not reached; 95\% CI: 40-53.5) and mean RFS was 44.9 months (95\% CI: 37.8-52.0). These patients were sequenced by using a 50 -gene panel composed of 1066 single-molecule molecular inversion probes (smMIPS) on an Illumina MiSeq sequencer ${ }^{8}$. Additional details pertaining to design of the panel and data analysis are described in Supplementary Methods (Supplementary Table 2). A total of 389 somatic mutations (including those occurring in NPM1 gene) were harbored by this cohort (Fig. 1). 

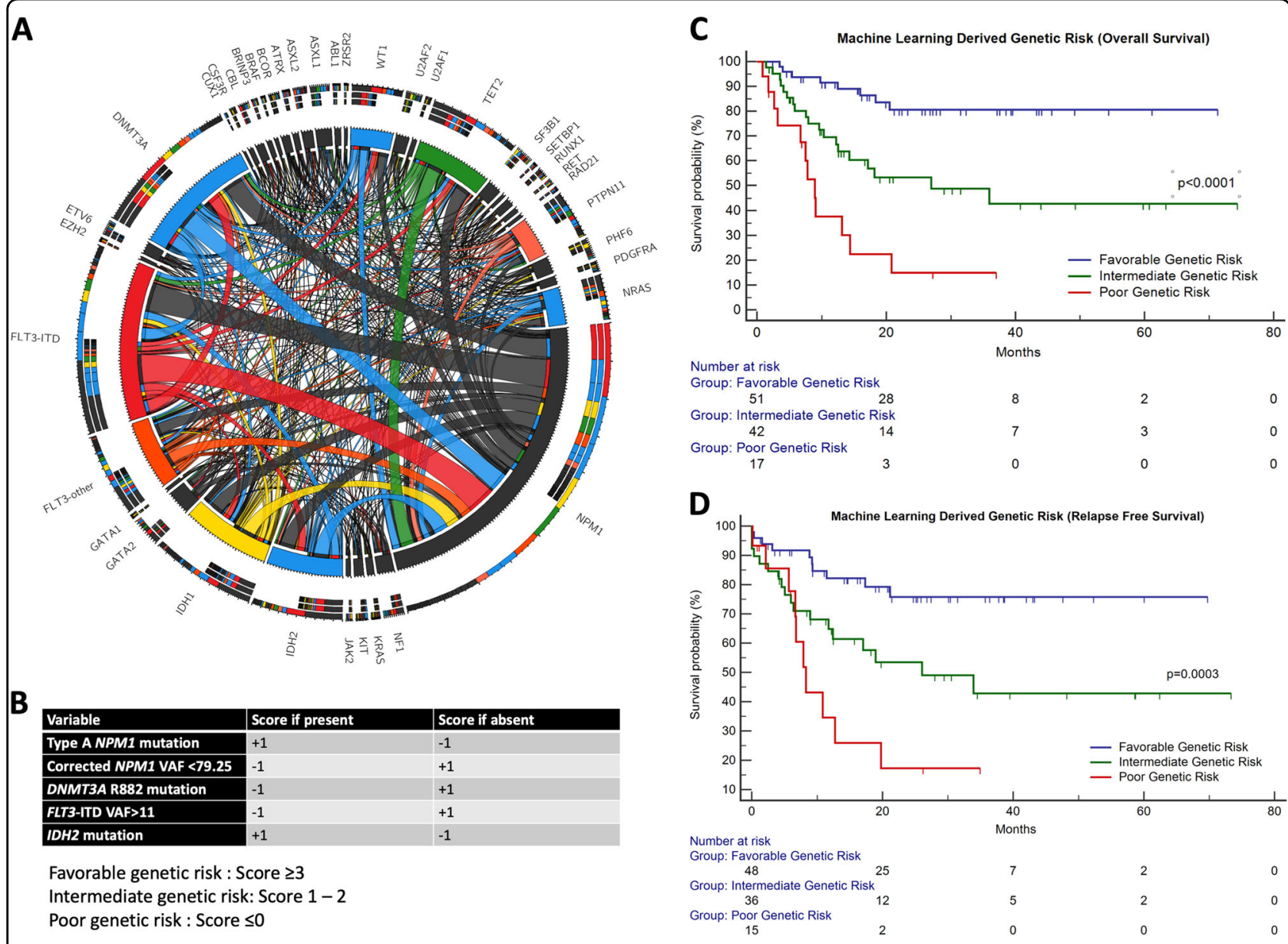

Fig. 1 A total of 389 somatic mutations were harbored among 110 patients. The above circos plot $(1 \mathrm{~A})$ highlights the genomic complexity of NPM1-mutated AML. Commonly occurring gene mutations are colored. The scoring system is detailed in panel $\mathbf{b}$. Clinical relevance of the machinelearning-derived genetic score for NPM1-mutated AML is also depicted here. The Kaplan-Meier plot in the top-right section (c) shows the clinical relevance of genetic risk when factored for overall survival (OS) and plot on the lower right (d) for relapse-free survival (RFS)

It is still unclear if NPM1 ${ }^{\text {mut }}$ AML in which the NPM1 gene per se is not a founder mutation has any different prognosis from the rest. To address this lacuna in the literature, we devised a new metric called corrected NPM1 VAF where we compute the NPM1 allelic abundance as a fraction of the largest VAF for that sample. For example, if NPM1 was the highest VAF for a given sample the corrected VAF was $100 \%$. Similarly, if NPM1 VAF was $40 \%$ and another variant was at $50 \%$, the corrected NPM1 VAF was $80 \%$. Based on receiver-operating characteristic (ROC) analyses, we determined that a corrected NPM1 VAF cutoff value of $\leq 79.25$ provided the optimal classification of patients as NPM1 VAF high or low. A similar ROC analysis was done for FLT3-ITD VAF levels where VAF levels were computed against OS to classify patients as FLT3-ITD VAF high or low. For FLT3-ITD VAF, a cutoff of 11 helped classify patients as high FLT3-ITD VAF (>11) and the rest as low FLT3-ITD VAF.
The performance characteristics of the ML model are depicted in supplementary data (Supplementary Tables 3 and 4, Supplementary Fig. 1). Based on these data, the top five variables most likely to predict a patient to be alive were high corrected NPM1 VAF, low FLT3-ITD VAF, presence of $I D H 2$ mutation, absence of DNMT3A R882 mutation, and type A NPM1 mutation. A final score for that case was devised as a sum total of the individual scores. This score is elaborated in Fig. 1. Measurable residual disease (MRD) was assessed by using multiparametric FCM (FCM-MRD). Out of 100 patients who were in morphological remission, post-induction FCM-MRD assessment was performed in 99. Of these FCM-MRD was detected in $27.1 \%$. The presence of FCM-MRD was predictive of an inferior OS $(p=0.007)$ and RFS $(p=0.01)$ as seen in Supplementary Data (Supplementary Fig. 2, Supplementary Table 5). A strong statistical correlation was observed between ML-derived 
Table 1 Prognostic significance of MRD, machinelearning-derived genetic risk in NPM1-mutated AML by univariate and multivariate Cox analysis

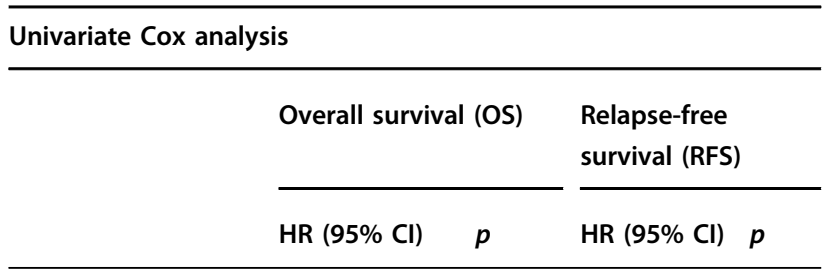

Machine-learning-derived genetic risk

$\begin{array}{llll}\begin{array}{lll}\text { Favorable } \\ \text { genetic risk }\end{array} & 1 & <0.0001 & 0.0003 \\ \text { Intermediate } & 3.54 & 2.71 \\ \text { genetic risk } & (1.81-6.94) & (1.36-5.38) \\ \text { Poor genetic risk } & 8.57 & 5.19 \\ & (2.84-25.86) & (1.76-15.27)\end{array}$

Post-induction FCM-MRD

$\begin{array}{lllll}\text { MRD negative } & 1 & 0.007 & 1 & 0.02 \\ \text { MRD positive } & 1.44(0.9-2.3) & & 1.48 & \\ & & & (1.0-2.18) & \end{array}$

\begin{tabular}{llll}
\hline Factors & HR & 95\% Cl & $\boldsymbol{p}$ \\
\hline Multivariate Cox analysis: overall survival & & \\
Intermediate genetic risk & 3.52 & $(1.42-8.78)$ & 0.0071 \\
Poor genetic risk & 7.94 & $(3.0-21.35)$ & $<0.0001$ \\
Post-induction FCM-MRD positive & 1.62 & $(1.1-2.37)$ & 0.015 \\
Multivariate Cox analysis: relapse-free survival & & \\
Intermediate genetic risk & 2.38 & $(1.07-5.31)$ & 0.03 \\
Poor genetic risk & 4.86 & $(1.98-11.97)$ & 0.0006 \\
Post-induction FCM-MRD positive & 2.05 & $(1.01-4.15)$ & 0.05 \\
\hline
\end{tabular}

MRD measurable residual disease, OS overall survival, RFS relapse-free survival, $H R$ hazards ratio, $\mathrm{Cl}$ confidence interval

genetic risk and post-induction FCM-MRD $((p=0.001)$, Supplementary Fig. 3).

Patients who were classified as poor genetic risk had an inferior OS and RFS as compared with patients in favorable and intermediate risk classes (Fig. 1c, d; Supplementary Table 5). The results of univariate and multivariate Cox analysis are seen in Table 1 . FCM-MRD as well as genetic risk were important determinants of outcome. On multivariate Cox analysis (Table 1), the presence of poor genetic risk was the most important independent factor when factored for OS as well as RFS.

Genetic scoring systems have been used systematically for precursor B lineage acute lymphoblastic leukemia by incorporating copy-number alteration and cytogenetics data with great success ${ }^{9}$. Rather than focusing on individual risk factors, we predicted that a combinatorial approach was most likely to yield relevant prognostic information. This is evident by good correlation of genetic risk classes with FCM-MRD as well as clinical outcome. This study, to the best of our knowledge, represents a novel application of ML to $N P M 1^{\text {mut }}$ AML. Our data indicate that this scoring system will be useful in identifying $N P M 1^{\text {mut }}$ AML patients who are at high risk of relapse and distinguishes them from patients who are at truly good risk. In our data set, poor genetic risk patients had a much shorter survival as compared with patients in favorable genetic risk category (Fig. 1; Supplementary Table 5). Such patients will require intensive postremission strategies, such as hematopoietic stem cell transplantation or experimental therapies.

Recently, Cappelli et al. in a large study on $N P M 1^{\text {mut }}$ AML demonstrated that DNMT3A R882 mutation was commonly seen in younger adults as compared with older patients ${ }^{10}$. Although our cohort is a young AML cohort, we found that these R882 mutations were almost equally distributed as compared with other DNMT3A mutations (15.5\% as compared with $16.4 \%)$. In addition, we found that DNMT3A R882 mutations are associated with inferior outcome as opposed to other DNMT3A mutations (Supplementary Fig. 4).

Dunlap et al. recently demonstrated that $I D H$ mutations in combination with $D N M T 3 A$ mutations predict for an inferior outcome ${ }^{11}$. However, the clinical relevance of $I D H$ mutations in AML is unclear due to conflicting data $^{4,11-13}$. Our data indicate that $I D H 2$ (in our data set limited to IDH2 R140 hotspot mutation) and NPM1 comutated AML is a favorable disease entity especially in the context of other variables in the genetic scoring system proposed by us (Supplementary Fig. 5).

High allelic fractions of recurrently mutated genes in AML such as FLT3 (namely FLT3-ITD) are associated with poor outcome ${ }^{14}$. Patel et al. described that high NPM1 VAF levels had an association with poor outcome $^{15}$. These findings were however refuted by another group $^{16}$. Rather than analyzing upfront VAF levels, we devised a new metric called corrected NPM1 VAF. Expectedly, cases where NPM1 is not the early clone are dominated by ARCH mutations (Supplementary Fig. 6), and this may be an additional factor contributing to poor outcome. In fact, patients with low corrected NPM1 VAF harbored higher frequencies of IDH1 mutations as compared with the rest (Supplementary Fig. 7). On factoring in the type of NPM1 mutation (type A or otherwise) based on existing literature, we determined that this was clinically relevant especially in the context of other MLderived variables ${ }^{17,18}$. 
To summarize, a supervised ML approach identified clinically important genomic aberrations in $N P M 1^{\text {mut }}$ AML. By using these data, we devise a scoring system that enables us to subclassify NPM1-mutated AML into three prognostic classes. We demonstrate a good correlation of this machine-learning-derived genetic score with FCM-MRD. Finally, we also show that ML-derived genetic risk classes have vastly differing outcomes, and these classes are independent predictors of clinical outcome. The limitations of our study include a relatively small cohort and retrospective analysis. The cutoffs for corrected NPM1 and FLT3-ITD VAFs in this study will only be approximate in nature, and given the variability of different NGS methodologies as well as sequencing platforms, are likely to change. The scoring system as well as these cutoffs should be validated prospectively by other groups.

\section{Acknowledgements}

We are grateful for the training imparted to Dr Nikhil Patkar in the field genome-sequencing technologies by Dr David Wu, Dr Stephen Salipante, and Dr Brent Wood. We would like to acknowledge the mentorship of Nikhil Patkar by Dr David Wu, Dr Stephen Salipante, and Dr Brent Wood in the Department of Laboratory Medicine, University of Washington, USA.

\section{Funding}

This work was supported by the Wellcome Trust/DBT India Alliance Fellowship [grant number IA/CPHI/14/1/501485] awarded to Dr Nikhil Patkar.

\section{Author details}

'Haematopathology Laboratory, ACTREC, Tata Memorial Centre, Navi Mumbai, Maharashtra, India. ${ }^{2}$ Biostatistics, ACTREC, Tata Memorial Centre, Navi Mumbai, Maharashtra, India. ${ }^{3}$ Adult Haematolymphoid Disease Management Group, Tata Memorial Centre, Mumbai, Maharashtra, India. ${ }^{4}$ Department of Cytogenetics, ACTREC, Tata Memorial Centre, Navi Mumbai, Maharashtra, India

\section{Conflict of interest}

The authors declare that they have no conflict of interest.

\section{Publisher's note}

Springer Nature remains neutral with regard to jurisdictional claims in published maps and institutional affiliations.

Supplementary information accompanies this paper at (https://doi.org/ 10.1038/s41408-019-0244-2).
Received: 11 July 2019 Revised: 16 September 2019 Accepted: 18 September 2019

Published online: 01 October 2019

\section{References}

1. Thiede, C. et al. Prevalence and prognostic impact of NPM1 mutations in 1485 adult patients with acute myeloid leukemia (AML). Blood 107, 4011-4020 (2006).

2. Patel, J. L. et al. Coexisting and cooperating mutations in NPM1-mutated acute myeloid leukemia. Leuk. Res. 56, 7-12 (2017).

3. Shlush, L. I. Age-related clonal hematopoiesis. Blood 131, 496-504 (2018).

4. Papaemmanuil, E. et al. Genomic classification and prognosis in acute myeloid leukemia. N. Engl. J. Med. 374, 2209-2221 (2016).

5. Libbrecht, M. W. \& Noble, W. S. Machine learning applications in genetics and genomics. Nat. Rev. Genet. 16, 321-332 (2015).

6. Kourou, K, Exarchos, T. P., Exarchos, K. P., Karamouzis, M. V. \& Fotiadis, D. I. Machine learning applications in cancer prognosis and prediction. Comput. Struct. Biotechnol. J. 13, 8-17 (2015).

7. Shouval, R. et al. Prediction of allogeneic hematopoietic stem-cell transplantation mortality 100 days after transplantation using a machine learning algorithm: A European Group for Blood and Marrow Transplantation Acute Leukemia Working Party Retrospective Data Mining Study. J. Clin. Oncol. 33, 3144-3151 (2015).

8. Hiatt, J. B., Pritchard, C. C., Salipante, S. J., O'Roak, B. J. \& Shendure, J. Single molecule molecular inversion probes for targeted, high-accuracy detection of low-frequency variation. Genome Res. 23, 843-854 (2013).

9. Moorman, A. V. et al. A novel integrated cytogenetic and genomic classification refines risk stratification in pediatric acute lymphoblastic leukemia. Blood 124, 1434-1444 (2014).

10. Cappelli, L. V. et al. DNMT3A mutations are over-represented in young adults with NPM1 mutated AML and prompt a distinct co-mutational pattern. Leukemia. https:/doi.org/10.1038/s41375-019-0502-0 (2019).

11. Dunlap, J. B. et al. The combination of NPM1, DNMT3A, and IDH1/2 mutations leads to inferior overall survival in AML. Am. J. Hematol. 94, 913-920 (2019).

12. DiNardo, C. D. et al. Characteristics, clinical outcome, and prognostic significance of IDH mutations in AML. Am. J. Hematol. 90, 732-736 (2015).

13. Paschka, P. et al. IDH1 and IDH2 mutations are frequent genetic alterations in acute myeloid leukemia and confer adverse prognosis in cytogenetically normal acute myeloid leukemia with NPM1 mutation without FLT3 internal tandem duplication. J. Clin. Oncol. 28, 3636-3643 (2010).

14. Gale, R. E. et al. The impact of FLT3 internal tandem duplication mutant level, number, size, and interaction with NPM1 mutations in a large cohort of young adult patients with acute myeloid leukemia. Blood 111, 2776-2784 (2008).

15. Patel, S. S. et al. High NPM1-mutant allele burden at diagnosis predicts unfavorable outcomes in de novo AML. Blood 131, 2816-2825 (2018).

16. Abbas, H. A. et al. NPM1 mutant variant allele frequency correlates with leukemia burden but does not provide prognostic information in NPM1mutated acute myeloid leukemia. Am. J. Hematol. 94, E158-E160 (2019).

17. Alpermann, T. et al. Molecular subtypes of NPM1 mutations have different clinical profiles, specific patterns of accompanying molecular mutations and varying outcomes in intermediate risk acute myeloid leukemia. Haematologica 101, e55-e58 (2016).

18. Pastore, F. et al. The NPM1 mutation type has no impact on survival in cytogenetically normal AML. PLoS One 9, e109759 (2014). 